\title{
The effect of early postnatal discharge from hospital for women and infants: a systematic review protocol
}

\author{
Eleanor Jones*, Beck Taylor, Christine MacArthur, Ruth Pritchett and Carole Cummins
}

\begin{abstract}
Background: The length of postnatal hospital stay has declined over the last 40 years. There is little evidence to support a policy of early discharge following birth, and there is some concern about whether early discharge of mothers and babies is safe. The Cochrane review on the effects of early discharge from hospital only included randomised controlled trials (RCTs) which are problematic in this area, and a systematic review including other study designs is required. The aim of this broader systematic review is to determine possible effects of a policy of early postnatal discharge on important maternal and infant health-related outcomes.
\end{abstract}

Methods/design: A systematic search of published literature will be conducted for randomised controlled trials, non-randomised controlled trials (NRCTs), controlled before-after studies (CBA), and interrupted time series studies (ITS) that report on the effect of a policy of early postnatal discharge from hospital. Databases including Cochrane CENTRAL, MEDLINE, EMBASE, CINAHL and Science Citation Index will be searched for relevant material. Reference lists of articles will also be searched in addition to searches to identify grey literature. Screening of identified articles and data extraction will be conducted in duplicate and independently. Methodological quality of the included studies will be assessed using the Effective Practice and Organisation of Care (EPOC) criteria for risk of bias tool. Discrepancies will be resolved by consensus or by consulting a third author. Meta-analysis using a random effects model will be used to combine data. Where significant heterogeneity is present, data will be combined in a narrative synthesis. The findings will be reported according to the preferred reporting items for systematic reviews (PRISMA) statement.

Discussion: Information on the effects of early postnatal discharge from hospital will be important for policy makers and clinicians providing maternity care. This review will also identify any gaps in the current literature on this topic and provide direction for future research in this area of study.

Systematic review registration: PROSPERO CRD42015020545

Keywords: Postnatal care, Early discharge, Length of stay

\section{Background}

The average length of postnatal stay in England has decreased over the last 40 years. In 1975, $32 \%$ of women were discharged within 3 days of giving birth compared to $91 \%$ of women in 2013-2014 [1, 2]. The proportion of women and babies who are discharged on the date of delivery has also increased. In 2005-2006, $16.5 \%$ of

\footnotetext{
* Correspondence: exj480@student.bham.ac.uk Institute for Applied Health Research, University of Birmingham, Edgbaston, Birmingham B15 2TT, UK
}

women were discharged on the same day that they gave birth compared to $20.3 \%$ of women in 2013-2014 [2]. Despite an increase in medical intervention during childbirth and more complex needs of women who become pregnant, there is some evidence to suggest that low-risk women and babies are being discharged from 4-6 h following birth $[3,4]$. The decline in postnatal stay in hospital is consistent with the USA, Australia and Canada and is considered to be primarily policy driven in efforts to accommodate a rising birth rate $[5,6]$. 
There is little data and some concern about whether early discharge of mothers and babies is safe. It has been suggested that early discharge from hospital leaves insufficient time for women and babies to establish breastfeeding and, as a result, leads to feeding-related problems [7]. In addition, it is argued that early discharge may increase the delay in the identification and treatment of maternal and infant morbidity [8, 9]. In contrast, others have suggested that early discharge from hospital creates opportunities for family-centred care, creates greater opportunities for families to bond in their home environment and is a safe and cost-effective way to provide postnatal care $[10,11]$.

The existing evidence on the effects of early postnatal discharge from hospital is inconclusive. The most recent Cochrane systematic review including 10 randomised controlled trials (RCTs) (involving 4489 women) compared early postnatal discharge with a standard length of stay. The pooled estimate of the included trials showed no statistically significant difference between early discharge and standard length of stay for infant readmission to hospital (relative risk (RR) $1.2995 \%$ CI 0.60-2.79) or other important outcomes [5]. One of the main limitations of this review is the methodological and clinical heterogeneity within included studies.

Firstly, the review authors used the definition of 'early discharge' given by each trial team, and these ranged from $12 \mathrm{~h}$ to 3.5 days postpartum [12-14]. As a result, early discharge in one trial was the equivalent of standard length of stay in another trial. Secondly, the definition of 'healthy women and infants' differed among trials where some studies excluded women with comorbidities such as diabetes and others did not [12, 13]. Finally, the trials had different co-interventions in the early discharge groups ranging from being monitored at home for the first $24 \mathrm{~h}$ after birth [15] to only having two home visits once discharged from hospital [12]. Statistical heterogeneity was found when data from the trials were pooled in meta-analysis, and this is likely due to the varying definitions of early discharge, differing cointerventions and populations which were not clinically comparable. As a result, it is difficult to draw meaningful conclusions about the impact of shortened or 'early' postnatal stay in hospital.

To look more specifically at the RCTs included in the existing systematic review, one RCT which included 2324 women found that infants were twice as likely to be readmitted to hospital in the first month postnatally if they were discharged early $(<48 \mathrm{~h})$ compared to a standard length of stay in hospital (>48 h) (RR $2.1495 \%$ CI 1.2-7.5). Although this trial is the largest of its kind, its validity and reliability were compromised by noncompliance in the allocated intervention (50 \% noncompliance in the intervention group), poor recruitment (only $20 \%$ of women eligible chose to take part) and a sample size which was not large enough to detect significant differences between the intervention and comparison groups. Other trials had similar methodological constraints [16-19]. Although an RCT is generally the best method to evaluate the effects of an intervention, in the context of evaluating early postnatal discharge from hospital, an RCT design is likely to be both problematic and impractical. This has discouraged researchers from conducting further RCTs to assess the effect of early discharge from hospital on infant or maternal morbidity.

To this end, several large retrospective cohort studies have been conducted looking specifically at maternal and infant readmission rates to hospital within 28 days of birth. More specifically, researchers have assessed infant readmission to hospital for jaundice, gastroenteritis, dehydration and poor weight gain [8, 20-23]. Researchers examining maternal readmission rates have looked specifically at readmissions for postpartum haemorrhage, retained products of conception, infection and postpartum psychosis [24, 25]. These causes of readmission are particularly relevant in exploring the effect of postnatal length of stay in hospital. It is suggested by some that these causes of readmission may reflect an inadequate assessment of readiness for discharge from hospital and could possibly be avoided if sufficient support is available in the early postnatal period $[5,8,12]$. However, several of these studies were conducted using routine hospital data and from healthcare insurance claims data and not all known confounding factors were measured or adjusted for in data analysis. It is not possible to infer any causal relationship between early discharge and infant morbidity using these types of studies alone.

Despite the existing literature available on early postnatal discharge from hospital, there is insufficient evidence to inform policy. Although there is an existing Cochrane review with clearly specified outcome measures [5], it is limited by significant clinical and methodological heterogeneity. In addition, it is clear that an RCT design in this context is compromised by poor recruitment and participant crossover. Taking this into consideration, this systematic review will address the same research questions and use the same objectives and outcome measures as the Cochrane review but will broaden the study design criteria to include both RCTs and quasi-experimental studies. In addition, to allow meaningful comparison across studies, this systematic review will further describe the clinical characteristics and discharge criteria for the women and infants included in primary studies and will explore the effect of clinical variation in subgroup analysis. Early discharge will be defined as $<48 \mathrm{~h}$ to reflect contemporary postnatal discharge practices [2]. To ensure rigour, the protocol 
for this systematic review has been guided by the PRISMA P checklist (Additional file 1).

The aim of this systematic review is to determine the effects of a policy of early postnatal discharge $(<48 \mathrm{~h})$ for women and infants. It will consider whether there is an association between early postnatal discharge and readmission to hospital. It is hypothesised that early postnatal discharge may increase maternal and infant utilisation of health services.

As guided by the Cochrane review [5], the primary objective of this systematic review is to assess how effective an early postnatal discharge policy is in terms of important maternal and infant health related outcomes.

Specific objectives are to identify whether a policy of early discharge is associated with:

- infant readmission to hospital;

- duration of infant readmission;

- attendance at hospital emergency departments for infant health issues;

- the number of contacts with health professionals regarding infant health issues postdischarge;

- maternal readmission to hospital;

- duration of maternal readmission;

- attendance at hospital emergency departments for maternal health issues;

- the number of contacts with healthcare professionals regarding maternal issues post discharge;

- maternal depression, anxiety and fatigue after the birth;

- occurrence of breastfeeding problems and/or duration of breastfeeding .

\section{Methods/design}

Criteria for considering studies for the review

\section{Types of studies}

Included studies must be either a RCT, nonrandomised controlled trial (NRCT), controlled before-after study (CBA) or interrupted time series study (ITS). As guided by Effective Practice and Organisation of Care group (EPOC), all RCTs and nonrandomised control trials must have at least two intervention and control sites [26]. All interrupted times series studies must have a clearly defined point in time when the early discharge policy occurred and a minimum of three data points before and three after the intervention. Because terminology used to describe study designs can be ambiguous, the EPOC study design algorithm will be used to help determine the study design [26].
If there is a paucity of the studies described above, a supplementary review will also include good-quality cohort studies located at a single site.

\section{Types of participants}

Women and infants who are considered 'fit for discharge' by their healthcare practitioners. Women may have given birth in a consultant led unit, co-located midwife led unit or stand-alone midwife led unit. It is recognised that there will be considerable variation in how 'fit for discharge' is defined and this will be explored in subgroup analysis.

\section{Types of interventions}

A policy of early discharge from hospital where 'early discharge' refers to discharge that is $<48 \mathrm{~h}$ following birth and earlier than standard care in the setting in which the intervention is implemented.

\section{Types of outcome measures}

Maternal and infant outcome measures will be guided by the Cochrane review [5].

\section{Primary infant outcomes}

- Proportion of infants readmitted to hospital within 7 days and within 28 days after birth

\section{Secondary infant outcomes}

- Proportion of infants readmitted for conditions which may be considered avoidable (including jaundice, dehydration, poor weight gain, gastroenteritis) in the first 28 days after birth

- Duration of infant readmission for infants admitted within 7 and within 28 days after birth

- Total duration of infant hospitalisation over the first 28 days

- Proportion of infants attending accident and emergency department within 7 days and within 28 days after the birth

- Proportion of infants seen by a health professional in a primary care setting for a health related problem in the first 28 days after birth

- Number of contacts with health professionals regarding the infant within 28 days after birth

\section{Primary maternal outcomes}

- Proportion of women readmitted for complications related to childbirth (including postpartum haemorrhage, retained products of conception, infection, postpartum psychosis) in the first 6 weeks after birth 
- Proportion of women breastfeeding (exclusively or partially) at 48 h, 6 weeks and 6 months after birth

- Proportion of women scoring above the cut off score indicating probable depression on a validated standardized instrument for measuring depression

\section{Secondary maternal outcomes}

- Duration of readmission for women readmitted after birth

- Total duration of maternal readmission hospitalisation

- Proportion of women attending hospital accident and emergency department

- Number of contacts with health professionals regarding maternal health issues within 4 weeks after birth

- Proportion of women reporting infant feeding problems within 4 weeks after birth

\section{Methods for identification of studies \\ Electronic searches}

The following electronic databases will be searched:

- CENTRAL (Cochrane Central Register of Controlled Trials)

- MEDLINE

- EMBASE

- CINAHL

- Science Citation Index

Searches for relevant literature on these databases will be done using a combination of free text and indexed terms (for example MeSH terms) and will be combined using Boolean operators. Search terms will be adjusted for each electronic database. Due to wide variation in the definitions of study designs, the search strategy will not be limited by study design type.

\section{Proposed search strategy}

Database: Ovid MEDLINE:

1. exp postnatal care/

2. postnatal.ti,ab.

3. postpartum period/

4. puerperium.ti,ab.

5. puerperium/

6. postpartum.ti,ab.

7. "length of stay"/

8. patient discharge/

9. discharge.ti,ab.

10.hospital stay*.ti,ab.

11.(early adj3 discharge).ti,ab.

12.patient readmission/ 13.readmission".ti,ab.

14.admission".ti,ab.

15.hospitalization/

16.outcome".ti,ab.

17.hospitali".ti,ab.

18.safety.ti,ab.

19.complication".ti,ab.

20.patient admission/

21.1 or 2 or 3 or 4 or 5 or 6

22.7 or 8 or 9 or 10 or 11

23.12 or 13 or 14 or 15 or 16 or 17 or 18 or 19 or 20

24.21 and 22 and 23

\section{Citation searching}

Citation searching using key papers which meet the inclusion criteria for the review will enable identification of further potentially eligible studies.

\section{Grey literature}

Grey literature will also be searched using Popline, Trip database and Web of Science conference proceedings citation index. The Department of Health, Royal College of Midwifery, Royal college of Obstetricians and Gynaecologists, National Childbirth trust and electronic theses (EThOS) websites will also be checked for relevant material. Internet searches will be performed in Google for any relevant unpublished studies.

No time, language or geographical restrictions will be applied.

\section{Searching other resources}

Reference lists of key full text articles included in the review will be checked to identify any potentially eligible studies.

\section{Data collection and analysis}

Selection of studies

All studies identified using the search strategy described will be screened for inclusion in the review using the eligibility criteria specifically designed to answer the research questions. Initially, a decision for potential inclusion of study will be based on titles and abstracts and where there is uncertainty about whether a study meets the eligibility criteria, over caution will be applied and the full article will be obtained for detailed assessment against the inclusion criteria.

Assessment of studies for potential inclusion will be performed independently and unblinded by two reviewers without consideration for the results. Any differences in opinions will be resolved through discussion until a consensus is reached. Any papers that are not unanimously excluded or included by both reviewers will be examined by both reviewers until an outcome is agreed. If necessary, a third person may be consulted. 
This process will ensure that bias is minimised when deciding whether to include or exclude certain studies.

It is possible that the eligibility criteria may need to be adjusted if it becomes apparent that relevant studies are being excluded from the review or irrelevant studies are being included. Reference management software EndNote will be used by one reviewer to keep a record of decisions made for each article in addition to paper form.

If there is a lack of information about a particular study, the authors will be contacted for clarification. Duplicate publications of research results will be identified and treated as a single study for the purpose of the review. In order to maintain transparency in the review study selection process, a flow diagram will portray the number of studies remaining in each stage of the selection process. In addition, a list of the studies excluded from the review will be documented as an appendix with brief reasons for exclusion for studies.

\section{Data extraction}

As guided by University of York Centre for Reviews and Dissemination [27], data extraction will be performed independently by two reviewers and disagreements will be noted and resolved by consensus among researchers or by arbitration by a third researcher. The EPOC data collection form has been adapted to answer the specific research questions for the systematic review. The form will enable data collection of potential confounding factors in primary studies and methods used to control confounding.

In line with good practice, data extraction forms will be piloted on a sample of included studies to ensure that all the relevant information is captured and that resources are not wasted on extracting data that is not required [27].

Assessment of methodological quality of included studies It is likely that the methodological rigour of the included studies for this review will vary considerably, and it is recognised that that poor-quality studies may obscure important intervention effects or lack of effects. Due to the inclusion of quasi-experimental studies which may be more susceptible to bias than RCTs, thorough assessment of the study quality will be required.

Two review authors will independently assess the risk of bias of the included studies using a descriptive approach and guided by the EPOC criteria for 'risk of bias' tool. Risk of bias for each study included in the review will be qualitatively summarised as part of the summary of findings tables.

The quality assessment tool will be piloted on a small selection of included studies. It is recognised that the quality assessment of studies may involve a degree of subjective judgement and any differences in opinion will be resolved through discussion.

In addition to this, if a supplementary review which includes good-quality cohort studies is necessary, the Strengthening the Reporting of Observational Studies in Epidemiology (STROBE) checklist for cohort studies [28] will be used as a quality assessment tool and risk of bias will be qualitatively summarised in a risk of bias table.

\section{Measures of intervention effect}

For RCTs, quasi-RCTs and CBAs, categorical data (e.g. proportion of infants readmitted) will be reported as RRs with $95 \%$ confidence intervals. Continuous data (e.g. duration of infant hospitalisations in the first 28 days) will be reported as mean difference (MD) and $95 \%$ confidence intervals. To aid the interpretation of clinical significance, the absolute risk may also be reported. Guidance will be sought from a statistician if meta-analysis of continuous outcomes requires standardisation across studies.

For ITS, as guided by Ramsey et al. [29], two effect sizes will be reported including the change in the outcome immediately after the introduction of the intervention and the change in slope of the regression lines.

\section{Unit of analysis errors \\ Methods for reanalysis of RCT and CBAs with potential unit of analysis error}

As guided by EPOC [30], cluster RCTs which have been analysed incorrectly by not accounting for clustering will be reanalysed if possible. If the unit of analysis error cannot be corrected, the effect size will be reported without a standard error and confidence interval as they are unlikely to be accurate.

\section{Methods of reanalysis for ITS comparisons with inappropriate analysis}

Where possible, as guided by EPOC, segmented time series regression will be used to reanalyse the data using methods described in Ramsay et al. [29].

\section{Dealing with missing data}

In line with good practice, authors of primary studies will be contacted to obtain missing data in order to appropriately describe the study results or perform metaanalysis [28]. The potential impact of missing data will be addressed in sensitivity analysis.

\section{Assessment of heterogeneity}

It is likely that there will be much diversity in the included studies, and the variety of study designs included in the review may result in significant heterogeneity. Forest plots will be visually examined and poor overlap 
between the confidence intervals will give an indication of statistical heterogeneity. The $x^{2}$ test of heterogeneity will help to determine whether differences between results are compatible with chance alone and the $I^{2}$ statistic will describe the percentage of variability in the effect estimates that can be attributed to heterogeneity rather than chance [31]. Where meta-analysis has been conducted, heterogeneity will also be explored through subgroup analysis.

\section{Data synthesis}

A summary of findings table of included studies will be produced for primary outcomes and will include key information concerning the type of study, number and characteristics of participants, interventions, outcomes and outcome measures.

Where appropriate, meta-analysis using a random effects model will be used to combine data. It is anticipated that there will be much diversity in the included studies and there will be considerable variation in what constitutes 'early discharge'. Where it is not appropriate to perform meta-analysis, included studies will be combined in a narrative synthesis and the results of the included studies will be combined in a forest plot with omission of the pooled estimate. To ensure the synthesis is a rigorous and transparent process, a narrative synthesis framework produced by Rodgers et al. [32] will be used. High-quality evidence will be given priority and results that are highly prone to bias will be interpreted with great caution.

\section{Sensitivity analyses}

Where meta-analysis is performed for primary outcomes, sensitivity analyses will explore the effect of

- study quality (by performing two meta-analyses, one which includes all eligible studies and another which only includes high-quality studies as defined by EPOC quality assessment criteria).

- missing data (by using a range of assumptions about the outcomes for participants lost to follow-up in the intervention versus the control arms varying from $100 \%$ intervention participants having a poor outcome to $0 \%$ ).

These sensitivity analyses have been decided a priori; however, further sensitivity analyses may be conducted if necessary.

\section{Subgroup analyses}

Subgroup analyses will investigate the effect of variables which may moderate the primary outcomes examined in the review and explore potential effect modifiers for primary outcomes. Using evidence from existing literature $[5,9,24]$, the following subgroups have been identified:

- Parity (primiparous women versus multiparous women)

- Gestation ( $<37$ weeks, 37-40 weeks, 40+ weeks)

- Timing of early discharge ( $\leq 12 \mathrm{~h}, \leq 24 \mathrm{~h}, \leq 48 \mathrm{~h}$ )

- Mode of delivery (vaginal delivery, operative vaginal delivery, elective caesarean, emergency caesarean)

- Co-intervention: early discharge accompanied by co-interventions (antenatal preparation or not, midwife home visits or not)

- Type of hospital delivered at (consultant led unit, co-located midwife led unit, stand-alone midwife led unit)

- Clinical characteristics of participants (low-risk women/infants, high-risk women/infants)

- 'Fit for discharge' criteria (for example, some primary studies may consider women who had blood loss of $>500 \mathrm{ml}$ or third degree perineal trauma as ineligible for early discharge and other studies may not).

\section{Discussion}

There is little evidence to support a policy early discharge from hospital. This systematic review aims to build on the work of the existing Cochrane review [5] by further describing the participant inclusion criteria and utilising a wider range of study designs to determine the effects of early postnatal discharge from hospital for mothers and infants. To reflect current postnatal practices, this systematic review will also further define early discharge as $<48 \mathrm{~h}$ after delivery. It is anticipated that this review will identify any gaps in the current literature on this topic and provide direction for future research in this area of study.

\section{Additional file}

Additional file 1: PRISMA-P 2015 checklist: recommended items to include in a systematic review protocol. Completed PRISMA-P 2015

checklist for systematic review protocol: the effects of early postnatal

discharge for women and term infants. (DOCX 16 kb)

\section{Abbreviations \\ CBA: controlled before-after studies; EPOC: Effective Practice and Organisation of Care; ITS: interrupted time series; MD: mean difference; NRCTs: non-randomised controlled trials; RCT: randomised controlled trials; $\mathrm{RR}$ : risk ratio.}

Competing interests

The authors declare that they have no competing interests. 


\section{Authors' contributions}

EJ, CC, BT and CM conceived the study. EJ wrote the first draft, and CC, BT, $\mathrm{CM}$ and RP revised the protocol. All authors read and approved the final manuscript.

\section{Acknowledgements}

We would like to acknowledge the contribution of Sue Bayliss for her assistance with the electronic search strategy. This study protocol presents independent research funded by the National Institute for Health Research (NIHR) Collaborations for Leadership in Applied Health Research and Care for West Midlands Programme (CLAHRC-WM). The views expressed are those of the authors and not necessarily those of the NHS, the NIHR or the Department of Health. This systematic review will form part of Eleanor Jones' PhD research, supervised by Dr Carole Cummins, Professor Christine MacArthur and Dr Beck Taylor.

\section{Received: 28 September 2015 Accepted: 19 January 2016}

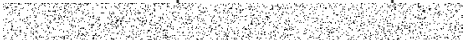

\section{References}

1. Richardson A, Mmata C. Maternity statistics England 2005-06. The Information Centre. 2007. http://www.hscic.gov.uk/catalogue/PUB01682/ nhs-mater-2005-2006-rep.pdf. Accessed 27 July 2015

2. Health and Social Care Information Centre. NHS Maternity Statistics in England 2013-2014. Health and Social Care Information Centre. 2015. http:// www.hscic.gov.uk/catalogue/PUB16725. Accessed 27 July 2015

3. The Royal College of Midwives. Pressure points: postnatal care planning. 2014. https://www.rcm.org.uk/get-involved/campaigns/pressure-points . Accessed 20 March 2015

4. Knight M, Kenyon S, Brocklehurst P, Neilson J, Shakespeare J, Kurinczuk J, et al. Saving lives, improving mothers' care-lessons learned to inform future maternity care from the UK and Ireland Confidential Enquires into Maternal Deaths and Morbidity 2009-2012. Oxford: National Perinatal Epidemiology Unit, University of Oxford; 2014

5. Brown S, Small R, Argus B, Davis PG, Krastev A. Early postnatal discharge from hospital for healthy mothers and term infants. Cochrane Database of Systematic Reviews. 2002; doi:10.1002/14651858.CD002958

6. Office for National Statistics. Births and deaths in England and Wales. 2011 http://www.ons.gov.uk/ons/rel/vsob1/birth-summary-tables-england-andwales/2011-final-/sb-births-and-deaths-in-england-and-wales-2011-final-. html. Accessed 20 March 2015.

7. Gupta P, Malhotra S, Singh D, Dua T. Length of postnatal stay in healthy newborns and re-hospitalization following their early discharge. Indian $J$ Pediatr. 2006; doi:10.1007/BF02859282

8. Danielsen B, Castles AG, Damberg CL, Gould JB. Newborn discharge timing and readmissions: California, 1992-1995. Pediatrics. 2000;106(1 l):31-9.

9. Lain $\mathrm{SJ}$, Roberts $\mathrm{CL}$, Bowen JR, Nassar N. Early discharge of infants and risk of readmission for jaundice. Pediatrics. 2015; doi:10.1542/peds.2014-2388

10. Waldenström U, Sundelin C, Lindmark G. Early and late discharge after hospital birth. Health of mother and infant in the postpartum period. Upsala Journal of Medical Sciences. 1987; doi:10.3109/ 03009738709178701.

11. Madden JM, Soumerai SB, Lieu TA, Mandl KD, Zhang F, Ross-Degnan D. Effects of a law against early postpartum discharge on newborn follow-up, adverse events, and HMO expenditures. N Engl J Med. 2002;347(25):2031-8.

12. Boulvain M, Perneger TV, Othenin-Girard V, Petrou S, Berner M, Irion O. Home-based versus hospital-based postnatal care: a randomised trial. BJOG: An International Journal of Obstetrics \& Gynaecology. 2004; doi:10.1111/j. 1471-0528.2004.00227.

13. Brooten D, Roncoli M, Finkler S, Arnold L, Cohen A, Mennuti M. A randomized trial of early hospital discharge and home follow-up of women having cesarean birth. Obstet Gynecol. 1994;84(5):832-8.

14. Yanover MJ, Jones D, Miller MD. Perinatal care of low-risk mothers and infants. New England Journal of Medicine. 1976; doi:10.1056/ NEJM197603252941306.

15. Bueno JAS, Romano MR, Teruel RG, Benjumea AG, Palacín AF, González CA, et al. Early discharge from obstetrics-pediatrics at the Hospital de Valme, with domiciliary follow-up. Am J Obstet Gynecol. 2005;193(3):714-26.

16. Carty EM, Bradley CF. A randomized, controlled evaluation of early postpartum hospital discharge. Birth: Issues in Perinatal Care. 1990;17(4): 199-204.
17. Winterburn S, Fraser R. Does the duration of postnatal stay influence breast-feeding rates at one month in women giving birth for the first time? A randomized control trial. J. Adv. Nurs. 2000; doi:10.1046/j.13652648.2000.01586

18. Gagnon AJ, Edgar L, Kramer MS, Papageorgiou A, Waghorn K, Klein MC. A randomized trial of a program of early postpartum discharge with nurse visitation. Am. J. Obstet. Gynecol. 1997; doi:10.1016/S0002-9378(97)80037-3

19. Thompson JF, Roberts $\mathrm{CL}$, Ellwood DA. Early discharge after childbirth: too late for a randomized trial? Birth. 1999; doi:10.1046/j.1523-536x.1999.00192

20. Kotagal UR, Atherton HD, Bragg E, Lippert C, Donovan EF, Perlstein PH. Use of hospital-based services in the first three months of life: impact of an early discharge program. Journal of Pediatrics. 1999; doi:10.1016/500223476(97)70351-2.

21. Liu S, Wen SW, McMillan D, Trouton K, Fowler D, McCourt C. Increased neonatal readmission rate associated with decreased length of hospital stay at birth in Canada. Can J Public Health. 2000;91 (1):46.

22. Madden JM, Soumerai SB, Lieu TA, Mandl KD, Zhang F, Ross-Degnan D. Length-of-stay policies and ascertainment of postdischarge problems in newborns. Pediatrics. 2004;113:42-9.

23. Young PC, Korgenski K, Buchi KF. Early readmission of newborns in a large health care system. Pediatrics. 2013;131:1538-44.

24. Liu S, Heaman M, Kramer MS, Demissie K, Wen SW, Marcoux S. Length of hospital stay, obstetric conditions at childbirth, and maternal readmission: a population-based cohort study. Am J Obstet Gynecol. 2002;187(3):681-7.

25. Ade-Conde JA, Alabi O, Higgins S, Visvalingam G. Maternal postnatal hospital readmission - trends and association with mode of delivery. Ir Med J. 2011;104:1.

26. Effective Practice and Organisation of Care (EPOC). What study designs should be included in an EPOC review and what should they be called? In: EPOC resources for review authors. Norweign Knowledge Centre for the Health Services, Oslo. 2015. http://epoc.cochrane.org/epoc-specificresources-review-authors. Accessed 17 April 2015.

27. Centre for Reviews and Dissemination. 1.3: Undertaking the review. In: Systematic reviews - CRD's guidance for undertaking reviews in health care. University of York: Centre for Reviews and Dissemination; 2009 p. 28-29.

28. von Elm E, Altman D, Egger M, Pocock S, Gotzsche P, Vandenbroucke J. The Strengthening the Reporting of Observational Studies in Epidemiology (STROBE) statement: guidelines for reporting observational studies. Lancet. 2007:370:1453-7.

29. Ramsay CR, Matowe L, Grilli R, Grimshaw JM, Thomas RE. Interrupted time series designs in health technology assessment: lessons from two systematic reviews of behavior change strategies. Int I Technol Assess Health Care. 2003;19(4):613-23.

30. Effective Practice and Organisation of Care (EPOC). Analysis in EPOC reviews. EPOC Resources for review authors. Oslo: Norweigian Knowledge for the Health Services; 2013. Available at: http://epoc.cochrane.org/epoc-specificresources-review-authors. Accessed 30 July 2015.

31. Higgins J, Green S. Cochrane Handbook for Systematic Reviews of Interventions. The Cochrane Collaboration; 2011.

32. Rodgers M, Sowden A, Petticrew M, Arai L, Roberts H, Britten N. Testing methodological guidance on the conduct of narrative synthesis in systematic reviews: effectiveness of interventions to promote smoke alarm ownership and function. Evaluation. 2009; doi:10.1177/1356389008097871.

\section{Submit your next manuscript to BioMed Central and we will help you at every step:}

- We accept pre-submission inquiries

- Our selector tool helps you to find the most relevant journal

- We provide round the clock customer support

- Convenient online submission

- Thorough peer review

- Inclusion in PubMed and all major indexing services

- Maximum visibility for your research

Submit your manuscript at www.biomedcentral.com/submit 